\title{
研究简报
}

\section{倾斜代数 $\mathrm{AR}$ 箭图中含直向模的 $\mathrm{DT}_{r}$ 轨道的个数*}

\author{
杜先能 \\ (安徽大学数学系, 合肥 230039)
}

\section{关键词 倾斜代数 直向模 $\tau$ 轨道 倾斜模}

设 $A$ 是一个连通的 $\operatorname{Artin}$ 代数. $\bmod -A$ 表示全体有限生成右 $A$ 模的范畴. $\Gamma_{A}$ 表示 $A$ 的 AR 箭图 Auslander-Reiten quiver). $\tau$ 表示 Auslander-Reiten 变换 DT,

一个 $A$ 模 $T_{A}$ 称为倾斜 (tilting) 模, 如果

(1) $T_{A}$ 的投射维数至多是 1 ;

(2) $\operatorname{Ext}_{A}^{1}(T, T)=0$;

(3) 存在正合序列 $0 \rightarrow A_{A} \rightarrow T_{A}^{\prime} \rightarrow T_{A}^{\prime \prime} \rightarrow 0$, 其中 $T_{A}^{\prime}, T_{A}^{\prime \prime}$ 是 $T_{A}$ 的直和项的直和.

如果 $A$ 是一个遗传代数, $T_{A}$ 是一个倾斜模, 则 $B=\operatorname{End}\left(T_{A}\right)$ 称为一个倾斜代数 ${ }^{[1]}$. 我们将 自由地使用文献[1]和[2] 中的记号和术语.

根据文献[1], 一个不可分解 $A$ 模 $M$ 称为直向的, 如果不存在循环 $M \stackrel{f_{1}}{\rightarrow} M_{1} \rightarrow \cdots \stackrel{f_{s}}{\rightarrow}$ $M_{s}=M$, 使得 $M_{i}$ 不可分解, $f_{i}$ 为非零非同构的映射. 直向模在代数表示论中占重要地位. 因 为预投射分支, 预内射分支以及一个倾斜代数的连接分支都完全由直向模组成.

在文献[3] 中, Skowronski-Smal $\phi$ 证明了: 如果 $\mathscr{C}$ 是 $\Gamma_{A}$ 的一个完全由直向模构成的连通分 支, 则 $\mathscr{C}$ 仅含有限多个 $\tau$ 轨道. 后来彭联刚和肖杰证明了 $\Gamma_{A}$ 至多有有限多个 $\tau$ 轨道包含直 向模 ${ }^{[4]}$. 在文献 [5] 中, Skowronski 也独立地证明了同样的结论. 本文将就倾斜代数给出含直 向模 $\tau$ 轨道个数的界. 我们证明了: 若 $A$ 为 tame 型时, 这个界不超过 $2 n$. 一般情形这个界 不超过 $3 n$. 这里 $n$ 为单 $A$ 模同构类的个数.

对倾斜代数 $\mathrm{AR}$ 箭图的连通分支形状的描述可参看文献[2] 和文献[6～9].

根据文献[6], 一个平移箭图 (translation quiver) 称为拟链 (quasi-serial), 如果它是稳定管 子 (stable tube) 或 $Z A_{\infty}$. 有关 sectional 路 (path)、射线插人 (ray insertion) 和余射线插人 (coray insertion) 等概念参见文献[1].

定理 1 设 $B=\operatorname{End}\left(T_{A}\right)$ 是一个倾斜代数. $\mathscr{C}$ 是 $\Gamma_{B}$ 的一个连通分支. 如果 $\mathscr{C}$ 由一个 拟链平移箭图经过射线插人或余射线插人得到, 则 $\mathscr{C}$ 的稳定部分 $\mathscr{C} s$ 不含直向模.

证 假设 $\mathscr{C}$ 由一个拟链平移箭图经射线插人得到 (对偶地可证另一情形). 如果 $\mathscr{C}$ 本 身就是一个拟链, 则 $\mathscr{C}$ 不含任何直向模. 如果 $\mathscr{C}$ 由一个稳定管子通过射线插人得到, 则 $\mathscr{C}$

1994-11-17 收稿

* 国家教委博士点基金资助项目 
至多有 $n$ 个 $\tau$ 轨道 ( $n$ 为非同构的单 $B$ 模的个数), 且其稳定部分 $\mathscr{C}_{s}=\varnothing$.

设 由 $Z A_{\infty}$ 经射线插人得到, 则存在 sectional 路：

$$
x_{1} \rightarrow x_{2} \rightarrow \cdots \rightarrow x_{s} \rightarrow \cdots \rightarrow x_{i} \rightarrow \cdots,
$$

其中 $x_{i}$ 属于不同的 $\tau$ 轨道. 因此存在一个整数 $m$, 使得 $x_{i}$ 是 $\tau$ 稳定的当且仅当 $i>m$. 我们可 设 $m>0$ (否则, $\ell=Z A_{\infty}$ ). 这样对于 $i \leqslant m$, 有 $x_{\mathrm{i}}$ 位于某个投射模的 $\tau$ 轨道. 因此 $\mathscr{C}$ 的稳定 部分 $\mathscr{C}_{s}$ 是连通的. 由于 $\mathscr{C}_{s}$ 含有无限多个 $\tau$ 轨道且没有有问循环, 根据文献 $[10,11]$, 存在没 有有问循环的无限箭图 $\Delta$, 使得 $\mathscr{C}_{s}=\boldsymbol{Z} \Delta$. 此时再对 ${ }_{s}$ 重复文献[5] 中引理 3 的证明, 我们知 道 $\ell_{s}$ 不含任何直向模. 证毕.

记 $r$ 为 $\Gamma_{B}$ 中含直问模的 $\tau$ 轨道的个数, $n$ 为不同构的单 $B$ 模的个数.

定理 2 设 $B=\operatorname{End}\left(T_{A}\right)$ 为倾斜代数, 则

$$
n \leqslant r \leqslant 3 n \text {. }
$$

证 由于 $B$ 为倾斜代数, 根据文献[11], 倾斜模 $T_{A}$ 确定了 $\bmod -B$ 中的一个分裂挠理论 $(Y(T), \mathscr{Y}(T))$. 设 $\mathscr{C}$ 为 $\Gamma_{B}$ 的一个连通分支, 如果 $\ell$ 为 $\Gamma_{B}$ 的连接分支 (connecting compo-

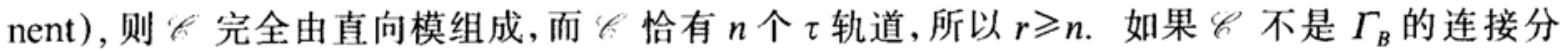
支, 则 $\mathscr{h}$ 完全包含在 $\mathscr{X}(T)$ 中且不含投射模, 或者 $\supsetneq$ 完全含在 $\mathscr{Y}(T)$ 中且不含内射模. 根据 文献[9], 如果 $\mathscr{C}$ 完全含在 $\mathscr{X}(T)$ 中, 则 $\mathscr{C}$ 或为预内射分支, 或为一个拟链, 或由一个拟链平 移箭图通过余射线插入得到. 如果 $\mathscr{C}$ 完全含在 $\mathscr{Y}(T)$ 中, 则 $\mathscr{C}$ 或为预投射分支, 或为一个拟 链, 或由一个拟链平移箭图经过射线插人得到.

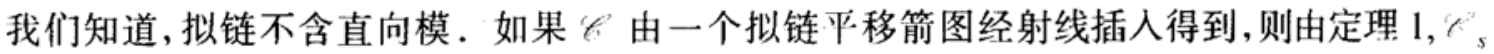
没有直向模. 从而 $\mathscr{C}$ 中的直向模只能位于投射模的 $\tau$ 轨道上. 如果 $\mathscr{C}$ 由一个拟链平移箭图 经余射线插人得到, 则由定理 1 知, $\mathscr{C}$ 中的直向模只能位于内射模的 $\tau$ 轨道. 但 $B$ 只有 $n$ 个 不可分解的投射 (内射) 模 (在同构意义下). 因此 $r \leqslant 3 n$. 证毕.

下面的定理给出了在 $A$ 为 tame 遗传代数时, $\Gamma_{B}$ 中含直向模的 $\tau$ 轨道个数的上下界.

定理 3 设 $A$ 为 tame 遗传代数, $B=\operatorname{End}\left(T_{A}\right)$ 为倾斜代数. 则 $n \leqslant r \leqslant 2 n$.

证 由于 $A$ 为 tame 遗传代数. 根据文献[2], 倾斜模 $T_{A}$ 只有以下几种可能性:

(i) $T_{A}$ 为预投射的，或 $T_{A}$ 为预内射的;

(ii) $T_{A}=T^{\prime} \oplus T^{\prime \prime}$, 其中 $T^{\prime} \neq 0$ 为预投射的, $T^{\prime \prime} \neq 0$ 为正则的;

(ii) $T_{A}=T^{\prime \prime} \oplus T^{\prime \prime \prime}$, 其中 $T^{\prime \prime} \neq 0$ 为正则的, $T^{\prime \prime \prime} \neq 0$ 为预内射的;

(iii) $T_{A}=T^{\prime} \oplus T^{\prime \prime} \oplus T^{\prime \prime \prime}$, 其中 $T^{\prime} \neq 0$ 为预投射的, $T^{\prime \prime}$ 为正则的, $T^{\prime \prime \prime} \neq 0$ 为预内射的.

由文献[1], 对情形 (i), 可以限制 $T_{A}$ 为预投射的. 这时 $B=\operatorname{End}\left(T_{A}\right)$ 为 tame concealed 代

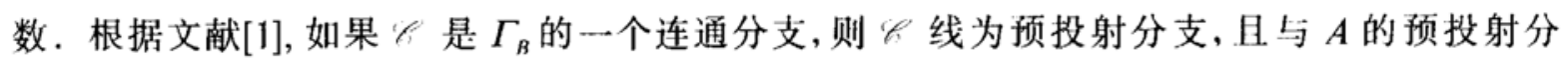
支有相同的轨道箭图; $\mathscr{C}$ 或为预内射分支, 且与 $A$ 的预内射分支有相同的轨道箭图; 或是 一个稳定管子. 因此在 (i) 的情形下, $\Gamma_{B}$ 恰有 $2 n$ 个 $\tau$ 轨道包含直向模 (我们始终假定 tame 型 不为有限型).

如果 $T_{A}$ 为 (ii) 的情形, 则 $B$ 是一个 tame concealed 代数的正则扩大 (regular enlargement). 如果 $\mathscr{C}$ 是 $\Gamma_{B}$ 的一个连道分支, 则 $\mathscr{Z}: "$ 为预投射的, 或为预内射的, 或为一个 稳定管子, 或为一个由稳定管子经过射线插人得到. 根据定理 $1, \Gamma_{B}$ 至多有 $2 n$ 个 $\tau$ 轨道包含 直问模. 
情形 (ii) ${ }^{\prime}$ 是情形 (ii) 的对偶.

如果情形 (iii) 成立, 则 $B$ 为有限表示型的, 从而 $B$ 为直向代数, 此时 $\Gamma_{B}$ 恰有 $n$ 个 $\tau$ 轨道包 含直向模. 综上各种情形, 我们得到 $n \leqslant r \leqslant 2 n$. 证毕.

注 由定理 3 的证明知道, $r$ 可以达到 $n$ 和 $2 n$. 下面的结论说明对非野 (Wild) 的倾斜代 数, $r$ 永远达不到 $3 n$.

定理 4 设 $B=\operatorname{End}\left(T_{A}\right)$ 为倾斜代数. 如果 $B$ 不是野型的, 则 $r<3 n$.

证 如果遗传代数 $A$ 为有限表示型的, 则 $B$ 也为有限表示型的, 此时 $r=n$. 如果 $A$ 为 tame 型的, 则由定理 $3, r \leqslant 2 n$.

现假设 $A$ 为野型的. 由于 $B$ 不为野型的, 所以倾斜模 $T_{A}$ 既有非零的预投射直和项, 又有 非零的预内射直和项. 设 $\mathscr{C}$ 是 $\Gamma_{B}$ 的由 $T_{A}$ 确定的连接分支, 则根据文献[1], $\mathscr{C}$ 既含有投射模 又含有内射模. 再由定理 2 的证明, 易知 $r<3 n$. 证毕.

下面的定理确定了某一类极小表示无限型代数含直向模的 $\tau$ 轨道的个数.

定理 5 设 $B$ 是一个极小表示无限的代数, 且具有预投射分支. 则 $\Gamma_{B}$ 中含直向模的 $\tau$ 轨道的个数是 $2 n$.

证 由于 $B$ 是极小表示无限型的, 且有预投射分支, 则根据文献[1], $B$ 是一个 tame concealed 代数, 或者 $B$ 是箭图 $\Delta: \overparen{:}$ 确定的路代数 (path algebra). 其中 $\Delta$ 有两个点, 至少有 两个箭向且所有箭向都指向同一个方向. 如果 $B$ 为前一情形, 则由定理 3 的证明即知结论正 确. 设 $B$ 为 $\Delta$ 确定的路代数. 由于 $B$ 为遗传代数, 所以 $B$ 恰有 $2 \times 2=4$ 个 $\tau$ 轨道包含直向 模.证毕.

致谢 衷心感谢导师刘绍学教授的关怀和指导. 衷心感谢张英伯教授、肖杰教授的有益 建议和关心。感谢邓邦明博士后的有益讨论.

\section{参考文献}

1 Ringel C M. Tame algebras and integral quadratic forms. LNM 1099, New York: Springer-Verlag, 1984

2 Happel D, Ringel C M. Construction of tilted algebras. LNM 903, New York: Springer-Verlag, 1981. 125 144

3 Skowronski A, Smal $\phi$ S O. Directing modules. J Algebra, 1992, 147: 137 146

4 Peng L, Xiao J. On the number of DT,-orbits containing directing modules. Proc Amer Math Soc, 1993, 118: $753 \sim 756$

5 Skowronski A. Regular Auslander-Reiten components containing directing modules. Proc Amer Math Soc, 1994, 120: $19 \sim 26$

6 Ringel C M. The regular components of Auslander-Reiten quiver of a tilted algebra. Chinese Ann Math, 1988, 9B: $1 \sim 18$

7 Kerner O. Tilting wild algebras. J London Math Soc, 1989, 39:29 47

8 Kerner O. Stable components of wild tilted algebras. J Algebra, 1991, 142:35 57

9 Liu S. The connected components of an Auslander-Reiten quiver of a tilted algebra. J Algebra, 1993, 161: $505 \sim 523$

10 Zhang Y. The structure of stable components. Can J Math, 1991, 43:652 672

11 Happel D, Preiser U, Ringel C M. Vinberg's characterization of Dynkin diagrams using subadditive functions with application to DT,-periodic modules. LNM 832, New York: Springer-Verlag, 1980. $280 \sim 294$

12 Happel D, Ringel C M. Tilted algebras. Trans Amer Math Soc, 1982, 274:399 443 